POS PROCEEDINGS

\title{
Impact of rotation on the weak s process
}

\author{
Urs Frischknecht ${ }^{* \dagger}$ \\ Department of Physics, University of Basel, Switzerland \\ E-mail: urs.frischknecht@unibas.ch
}

\section{Raphael Hirschi}

Astrophysics Group, University Keele, UK

and Institute for the Physics and Mathematics of the Universe, University of Tokyo, Japan

\section{Thomas Rauscher}

Department of Physics, University of Basel, Switzerland

\section{Friedrich-Karl Thielemann}

Department of Physics, University of Basel, Switzerland

\begin{abstract}
The weak s process takes place in massive stars and it produces the majority of s-only isotopes in the atomic mass range from 60 to 90 . This process is qualitatively well understood. However, there are still large uncertainties remaining on the quantitative side. Rotation has a strong effect on the stellar structure and mixing, but its impact on the s process has not been studied yet. We implemented an extended and flexible reaction network inside the Geneva stellar evolution code (GENEC) to be able to study the influence of rotation on the s process. For a star with a particular initial mass and composition rotation increases the He core size and the central temperature enhancing the s-process efficiency during core helium burning. In turn the C-shell contribution is reduced since more ${ }^{22} \mathrm{Ne}$ has already been burnt during He-burning. Mixing induced by rotation also affects the contribution of the He-burning shell, since it leads to the production of primary ${ }^{14} \mathrm{~N}$ and primary ${ }^{22} \mathrm{Ne} .{ }^{22} \mathrm{Ne}$ and ${ }^{4} \mathrm{He}$ can again be transported to regions with higher temperatures below the convective He-shell, where ${ }^{22} \mathrm{Ne}(\alpha, n)$ becomes an efficient neutron source. To investigate the influence of reaction rate uncertainties besides the uncertainties of stellar structure and mixing, we have developed a one-zone post-processing network including Monte Carlo variations of the rates.
\end{abstract}

11th Symposium on Nuclei in the Cosmos, NIC XI

July 19-23, 2010

Heidelberg, Germany

* Speaker.

${ }^{\dagger}$ A footnote may follow. 


\section{Introduction}

The weak s process in massive stars is qualitatively well understood (see e.g. [1-3], but there are still uncertainties on the nuclear as well as on the stellar model side. At the start of helium burning ${ }^{14} \mathrm{~N}$ is converted to ${ }^{22} \mathrm{Ne}$. The s process takes place at the end of helium burning when the temperatures are high enough to run ${ }^{22} \mathrm{Ne}(\alpha, n)^{25} \mathrm{Mg}$ efficiently. Consequently s-process yields from central helium burning increase with increasing stellar mass. In C-shell burning again ${ }^{22} \mathrm{Ne}(\alpha, n){ }^{25} \mathrm{Mg}$ is the main neutron source, its strength being limited by the amount of ${ }^{22} \mathrm{Ne}$ left over from helium burning. Therefore weak s process is a secondary process in the standard case of non-rotating stars, because of the need for $\mathrm{CNO}$ and Fe in the initial stellar composition. What might stellar rotation change? Rotation increases stellar core masses (see e.g. [4]) and it enables mixing in otherwise radiation dominated unmixed zones in stellar interiors. This leads to the production of primary ${ }^{14} \mathrm{~N}$ and ${ }^{22} \mathrm{Ne}$ [5]. As a consequence the additional amount of neutrons released could boost the weak s process in particular at low Z [6].

\section{Impact of rotation on weak s process}

We calculated two $25 \mathrm{M}_{\odot}$ models at solar metallicity to study the effects of rotation on the s process. One model was calculated without and the other one with rotation. A typical rotation rate with $v_{\text {ini }} / v_{\text {crit }}=0.4$ was chosen for the model including rotation, corresponding to an average velocity of about $240 \mathrm{~km} \mathrm{~s}^{-1}$ on the main sequence. As initial composition we used the solar chemical composition of [7]. With the Geneva stellar evolution code (GENEC) [8], we followed the evolution of these stars until after central Ne burning. We implemented the Basel Network (BasNet, used before extensively in astrophysical nucleosynthesis calculations, see e.g. [9-11]) inside GENEC, which enabled us to follow the s-process network coupled with the stellar structure. For these calculations a network including 613 nuclei was used, similar to the one adopted by [3].

Inclusion of rotation induces slightly lower densities but higher temperatures at the center of the star. The higher temperatures lead to an earlier activation of ${ }^{22} \mathrm{Ne}(\alpha, n)$. Therefore more ${ }^{22} \mathrm{Ne}$ is burned in He burning and less left over for C-shell burning. As mentioned earlier, the rotating model has a larger convective He core. In our models the core size was different by $\approx 0.5 \mathrm{M}_{\odot}$ when s process occured, meaning that a larger mass is affected by s process due to rotation. The appearance of shear instabilities is another effect of rotation affecting s process in two ways. First, in non-convective regions above the $\mathrm{He}$ core the additional mixing transports freshly produced ${ }^{12} \mathrm{C}$ into $\mathrm{H}$-rich layers where it is converted into ${ }^{14} \mathrm{~N}$ by ${ }^{12} \mathrm{C}(p, \gamma){ }^{13} \mathrm{~N}\left(\beta^{+}\right){ }^{13} \mathrm{C}(p, \gamma){ }^{14} \mathrm{~N}$. This primary nitrogen is then converted into ${ }^{22} \mathrm{Ne}$ and is available as neutron source mainly in Heshell burning. Second, in our models the bottom of the convective He shell is not hot enough to burn ${ }^{22} \mathrm{Ne}$ efficiently, but in the rotating model the layers just below the convective zone are also mixed although on a longer timescale. The downward mixing of helium and neon boosts the $\mathrm{s}$ process in He shell and increases the He-shell contribution to the final s-process yields strongly.

Figure 1 displays abundance profiles of different isotopes for both models. ${ }^{70} \mathrm{Ge}$ (orange dotted line, diamond) and ${ }^{88} \mathrm{Sr}$ (red dashed line, cross) are plotted as indicators for the weak s process. The level of ${ }^{22} \mathrm{Ne}$ (green dashed line, filled circle) in the rotating model is considerably higher, consisting of primary and secondary neon, whereas without rotation only secondary neon is found 
in He shell. The enhanced s-process activity in He shell appears as a bump in the ${ }^{70} \mathrm{Ge}$ and ${ }^{88} \mathrm{Sr}$ profiles of the rotating model at around $6 \mathrm{M}_{\odot}$.
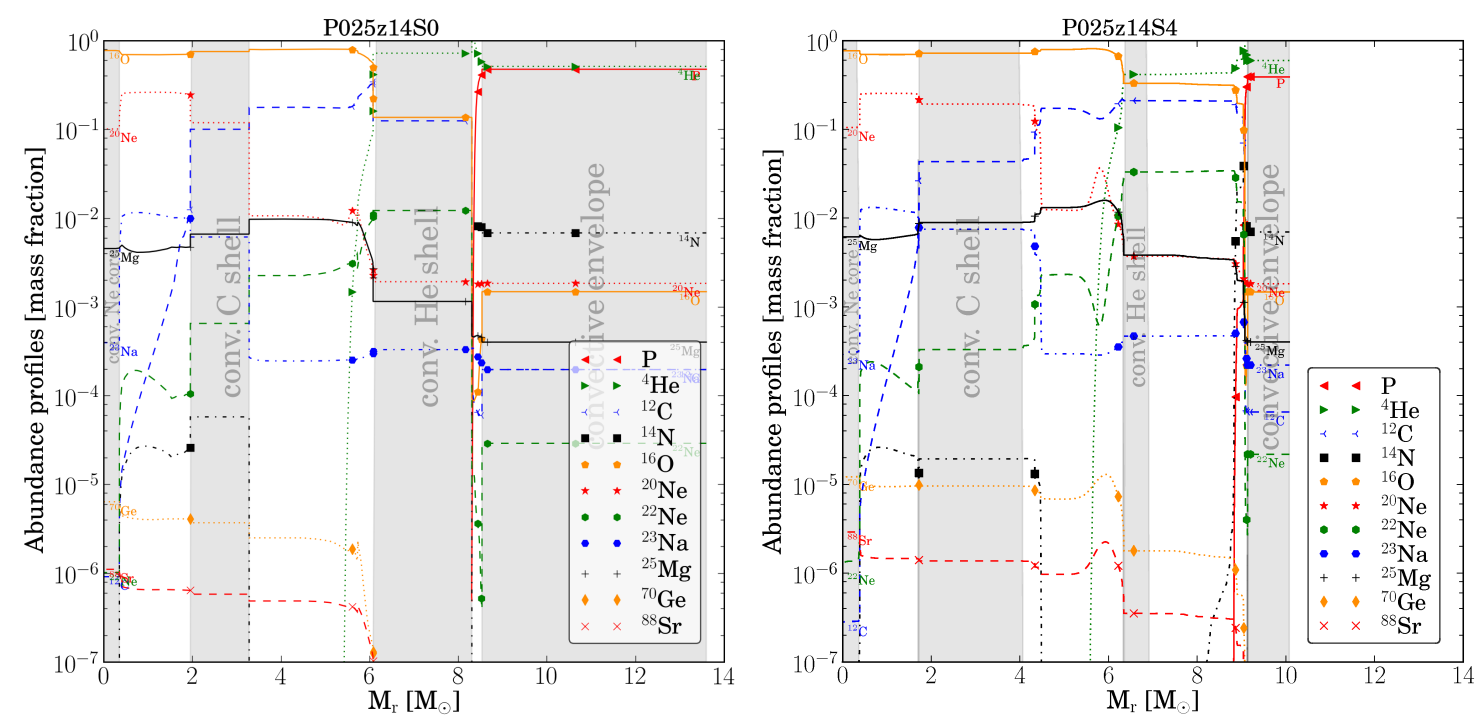

Figure 1: Abundance profiles (mass fraction versus mass) of the non-rotating (left) and rotating (right) $25 \mathrm{M}_{\odot}$ models during central Ne burning.

The s-process yields were estimated for both models by integrating from the bottom of the carbon shell up to the surface. Isotopes in the range of weak s process show in the rotating model higher yields by a factor 3 to 10 . The contribution to the total weak s-process yields from He shell increased from below $10 \%$ to over $30 \%$. The profile of weak s process (Y vs. A), did not change a lot due to rotation, since the s process is limited by the neutron source and not by the iron seeds at solar metallicity. The situation is reversed at low metallicities. Our models of rotating low $\mathrm{Z}$ stars show that a large production of primary ${ }^{22} \mathrm{Ne}$ is possible, even at low Z [5, 6]. This increases the ${ }^{22} \mathrm{Ne}$ to ${ }^{56} \mathrm{Fe}$ ratio and the neutron to seed ratio. This implies that in massive stars at low $\mathrm{Z}$ isotopes up to $\mathrm{Ba}$ or even heavier may be produced. The essential question will then be: how much primary ${ }^{22} \mathrm{Ne}$ can be produced by mixing induced by rotation on low metallicity stars? We will try to answer this question in our future work.

\section{Nuclear reaction rate sensitivity study}

Mainly the reaction rates including nuclei far from stability have large uncertainties. The astrophysical nucleosynthesis processes including such rates suffer therefore uncertainties accordingly. Such processes are e.g. p process, $r$ process, etc. It is therefore useful to have a tool allowing the investigation of the combined effect of all rate uncertainties using Monte Carlo methods. This has been done already in Astrochemistry [12] and astrophysical nucleosynthesis calculations (see e.g. $[13,14])$. But most of the previous sensitivity studies were based on manual rate by rate variation.

We have developed a one-zone post-processing reaction network code including Monte Carlo techniques for reaction rate variations. It can follow astrophysical trajectories (temperature, density) and allows us to investigate the sensitivity of different astrophysical processes on reaction rate 
uncertainties. The reaction rates can be varied in different ways, for instance multiplied by a factor which is log-normal or normal distributed around one, i.e. around the standard rate.

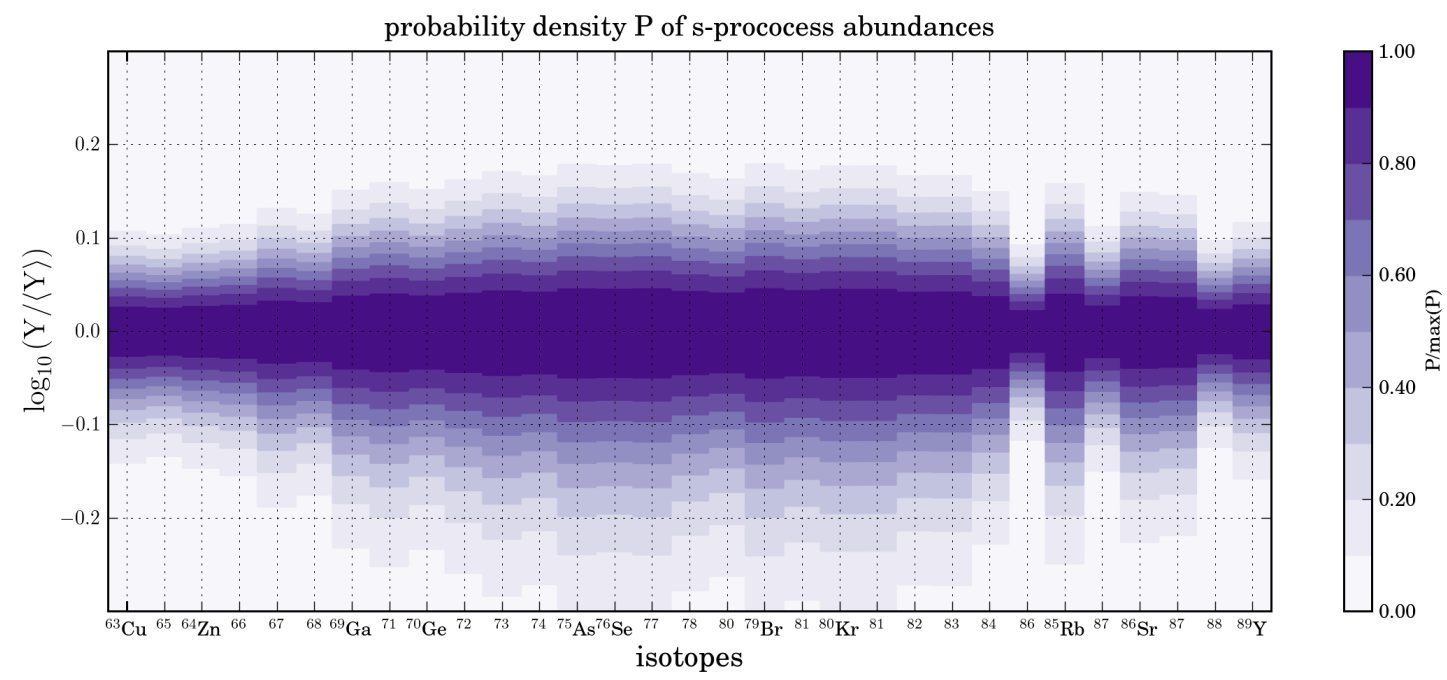

Figure 2: Normalised probability density function of s-process number abundances Y. For this calculation all $(\mathrm{n}, \gamma)$-rates were allowed to vary between the upper and lower limits, which were in this case $\pm 20 \%$ of the standard rates (KADoNiS, www.kadonis.org). For the repeated calculations a trajectory from a $25 \mathrm{M}_{\odot}$ model was taken and chosen in a way that the ${ }^{22} \mathrm{Ne}$ burned is equal in the trajectory and the stellar model. In this particular calculation, 266 rates were varied and the trajectory was sampled ten thousand times.

We investigated first as a test case the sensitivity of s process in He burning to uncertainties in $(\mathrm{n}, \gamma)$-rates. The abundance uncertainties after He burning are shown in Fig. 2. They grow from iron onwards up to Se. This is expected for weak s process, since the low neutron densities make the abundance of an isotope in this mass range dependent on the chain of $n$-captures and $\beta$-decays along the valley of stability from iron up to that particular isotope. This leads to a more or less linear behaviour of the uncertainties in s-process isotopes and makes it a good test case for such a Monte Carlo network.

\section{Acknowledgments}

This work was supported by grants of the Royal Society (IJP grant JP090091) and the Swiss National Science Foundation.

\section{References}

[1] M. Pignatari, R. Gallino, M. Heil, M. Wiescher, F. Käppeler, F. Herwig, and S. Bisterzo, The Weak s-Process in Massive Stars and its Dependence on the Neutron Capture Cross Sections, ApJ 710 (Feb., 2010) 1557-1577.

[2] L. S. The, M. F. El Eid, and B. S. Meyer, s-process nucleosynthesis in advanced burning phases of massive stars, ApJ 655 (Feb., 2007) 1058-1078, [arXiv : astro-ph/ 0609788 ].

[3] L. S. The, M. F. El Eid, and B. S. Meyer, A new study of s-process nucleosynthesis in massive stars, ApJ 533 (Apr., 2000) 998-1015. 
[4] R. Hirschi, G. Meynet, and A. Maeder, Yields of rotating stars at solar metallicity, A\&A 433 (Apr., 2005) 1013-1022, [arXiv:astro-ph/ 0412454 ].

[5] R. Hirschi, Very low-metallicity massive stars: pre-sn evolution models and primary nitrogen production, A\&A 461 (Jan., 2007) 571-583, [arXiv: astro-ph/ 0608170 ].

[6] M. Pignatari, R. Gallino, G. Meynet, R. Hirschi, F. Herwig, and M. Wiescher, The s-Process in Massive Stars at Low Metallicity: The Effect of Primary ${ }^{14} N$ from Fast Rotating Stars, ApJ 687 (Nov., 2008) L95-L98, [0 810 . 0182].

[7] M. Asplund, N. Grevesse, and A. J. Sauval, The Solar Chemical Composition, Cosmic Abundances as Records of Stellar Evolution and Nucleosynthesis (Sep., 2005) 25-+.

[8] P. Eggenberger, G. Meynet, A. Maeder, R. Hirschi, C. Charbonnel, S. Talon, and S. Ekström, The Geneva stellar evolution code, Ap\&SS 316 (Aug., 2008) 43-54.

[9] C. Fröhlich, G. Martínez-Pinedo, M. Liebendörfer, F. K. Thielemann, E. Bravo, W. R. Hix, K. Langanke, and N. T. Zinner, Neutrino-induced nucleosynthesis of a $>64$ nuclei: The vp-process, Physical Review Letters 96 (Apr., 2006) 142502, [arXiv: astro-ph/0511376].

[10] W. R. Hix and F. K. Thielemann, Computational methods for nucleosynthesis and nuclear energy generation., Journal of Computational and Applied Mathematics 109 (Sep., 1999) 321-351, [arXiv:astro-ph/9906478].

[11] W. D. Arnett and F. K. Thielemann, Hydrostatic nucleosynthesis. $i$ - core helium and carbon burning., ApJ 295 (Aug., 1985) 589-619.

[12] V. Wakelam, F. Selsis, E. Herbst, and P. Caselli, Estimation and reduction of the uncertainties in chemical models: application to hot core chemistry, A\&A 444 (Dec., 2005) 883-891, [arXiv:astro-ph/0509194].

[13] J. A. Stoesz and F. Herwig, Oxygen isotopic ratios in first dredge-up red giant stars and nuclear reaction rate uncertainties revisited, MNRAS 340 (Apr., 2003) 763-770, [arXiv:astro-ph/0212128].

[14] A. Parikh, J. José, F. Moreno, and C. Iliadis, The sensitivity of nucleosynthesis in Type I X-ray bursts to thermonuclear reaction-rate variations, New A Rev. 52 (Oct., 2008) 409-411, [0 806.2975 ]. 